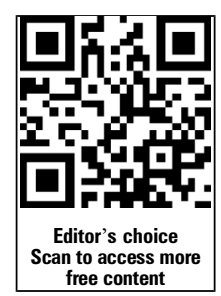

Neonatal Intensive Care Unit, St. Peter's Hospital, Ashford and St. Peter's Hospitals NHS Foundation Trust, Chertsey, UK

\section{Correspondence to} Dr Peter Reynolds, Neonatal Intensive Care Unit, St. Peter's Hospital, Ashford and St. Peter's Hospitals NHS Foundation Trust, Guildford Road, Chertsey, Surrey KT16 OPZ, UK; peter.reynolds@asph.nhs.uk

Received 2 September 2015 Revised 25 November 2015 Accepted 5 December 2015 Published Online First 5 January 2016

\section{SLinked}

- http://dx.doi.org/10.1136/ archdischild-2015-310269

CrossMark

To cite: Reynolds $P$, Leontiadi S, Lawson T, et al. Arch Dis Child Fetal Neonatal Ed 2016;101: F284-F287.

\title{
Stabilisation of premature infants in the delivery room with nasal high flow
}

\author{
Peter Reynolds, Stamatina Leontiadi, Tracy Lawson, Tosin Otunla, Olayinka Ejiwumi, \\ Nicola Holland
}

\begin{abstract}
Objective This was a pilot study to determine the feasibility of using nasal high flow (nHF) (also known as heated humidified high-flow nasal cannula) for stabilisation of babies born at $<30$ weeks gestation in the delivery room (DR) and transfer to the neonatal intensive care unit (NICU).

Design Observational study.

Setting Single-centre NICU.

Patients Infants born at $<30$ weeks gestation.

Interventions Stabilisation and transfer to NICU using $\mathrm{nHF}$.
\end{abstract}

Main outcome measures Feasibility of stabilisation as defined by successful transfer and clinical measures of stability at admission to NICU including oxygen

requirement, temperature, requirement for surfactant and inotrope use within $72 \mathrm{~h}$ of delivery.

Results Twenty-eight babies were enrolled after written parental consent had been obtained. 25/28 were successfully stabilised in the DR and transferred to the NICU on nHF. The average admission temperature for babies transferred on $\mathrm{nHF}$ was $36.9^{\circ} \mathrm{C}$ and the average inspired oxygen at admission was $29 \%$. Less than half $(48 \%)$ required surfactant and $60 \%$ were still on $\mathrm{nHF}$ $72 \mathrm{~h}$ after admission. 1 baby received inotropes.

Conclusions Our study suggests that using nHF for stabilisation of premature infants in the DR and subsequent transfer to NICU is feasible.

Clinical trial registration number NCT01991886.

\section{INTRODUCTION}

Stabilisation of preterm babies using nasal continuous positive airway pressure (NCAP) in the delivery room (DR) is recommended by international guidelines, ${ }^{12}$ with evidence of improved outcomes compared with routine intubation and ventilation. ${ }^{3} 4$ Selective administration of surfactant after stabilisation is also associated with improved outcomes. ${ }^{5}$ The use of nasal high flow (nHF) is increasingly established for the postextubation management of preterm babies, with several large studies demonstrating that it is generally as effective as nasal CPAP for postextubation support ${ }^{6-8}$ and may offer further advantages such as ease of use, greater comfort and reduction in nasal trauma. ${ }^{9}$ In our neonatal intensive care unit (NICU) we have been using nHF (Vapotherm Precision Flow) for noninvasive ventilation, as a replacement for nCPAP for $>8$ years. Our experience led us to hypothesise that $\mathrm{nHF}$ might be suitable for DR stabilisation and respiratory support immediately after birth. ${ }^{2}$ Given the paucity of literature on the use of nHF in the DR, it was not known if nHF would be straightforward to use in the DR setting, if it would be

\section{What is already known on this topic?}

Delivery room stabilisation of preterm infants using nasal continuous positive airway pressure (NCPAP) improves outcomes.

- Nasal high flow (nHF) is not inferior to NCPAP for postextubation management of neonates.

- nHF has gained popularity due to ease of use, effectiveness and patient comfort.

\section{What this study adds?}

It is feasible to stabilise premature babies in the delivery room (DR) using nasal high flow (nHF).

- Stabilisation on $\mathrm{nHF}$ may reduce rates of DR intubation and surfactant administration.

- The clinical stability of the well preterm infant may be evident soon after birth.

tolerated in the first few minutes after birth, nor if babies could be stabilised and moved to the NICU using nHF support. We, therefore, conducted a pilot study to establish the feasibility of using $\mathrm{nHF}$ in the DR to stabilise and transfer preterm infants born at 30 weeks gestation or less.

\section{MATERIALS AND METHODS}

\section{Study design}

This was an observational study to determine the feasibility (defined as 'The state or degree of being easily or conveniently done ${ }^{10}$ ) of the use of $\mathrm{nHF}$ in the stabilisation and transfer of preterm infants. We used quantitative measures to determine feasibility including transfer to NICU on nHF, evidence of clinical stability on admission to NICU-temperature and oxygen requirement and need for further stabilisation (eg, intubation and ventilation, surfactant administration and use of inotropes). Staff comments were invited.

\section{Setting}

The study was carried out in the NICU at St. Peter's Hospital, Chertsey, Surrey, UK between January 2014 and March 2015. Prior to this study, our normal practice was that babies born at $<26$ weeks gestation were electively intubated in the DR and received prophylactic surfactant (Curosurf 100$200 \mathrm{mg} / \mathrm{kg})^{2}{ }^{2}$ Above 26 weeks gestation intubation would be at the discretion of the attending clinician. 
Intubated babies would be transferred on intermittent positive pressure ventilation (IPPV), with early extubation to nHF if possible. Non-intubated babies would be stabilised and transferred to NICU on the resuscitaire using either CPAP or IPPV by face mask using the resuscitaire gas supply and nHF started when in the NICU. The majority of babies followed these pathways. Rescue surfactant would normally be given if the $\mathrm{FiO}_{2}$ was persistently $\geq 0.4$ although clinical judgement of work-of-breathing and rate of rise of $\mathrm{FiO}_{2}$ would be taken into account. Dopamine would be started as the first line inotrope if the mean blood pressure was lower than $20 \mathrm{~mm} \mathrm{Hg}$, or was persistently lower than the gestational age with evidence (clinical or biochemical) of poor perfusion.

\section{Prestudy practice on our NICU}

Our routine practice for the DR management of babies born at $<30$ weeks gestation during the year preceding this study was noted for comparison. Of 52 babies born in 2013, 73\% were intubated in the DR ( $100 \%$ if born at $<26$ weeks), with all intubated babies receiving surfactant in the DR. One planned and three unplanned extubations occurred prior to transfer to NICU, and $15 / 34(44 \%)$ were then electively extubated within $24 \mathrm{~h}$ on NICU. We have a long-standing policy of early extubation and non-invasive ventilation to nHF. 5/52 babies were still ventilated at $72 \mathrm{~h}$ with only two babies receiving inotropes. There were three deaths. We do not use nCPAP routinely on our NICU for stabilisation or postextubation management and thus nCPAP was not a rescue option in the protocol for this study.

\section{Participants}

Babies were enrolled if they delivered between $23+0$ and 29 +6 weeks gestation and written parental consent had been obtained prior to delivery. For twin pregnancies, parents were counselled that, as there was only one mobile nHF device, we would apply nHF to the first twin initially if appropriate, with the option to apply nHF to the second twin depending on the progress of twin one and time permitting.

\section{Delivery room nHF}

In order to carry out this study, a mobile apparatus to deliver $\mathrm{nHF}$ was designed and constructed. Vapotherm constructed a prototype to our specifications, based on their Precision Flow high-flow device, which could be taken to each delivery and then moved with the baby to NICU, and could be operated with and without mains electricity and piped oxygen and air supplies. A gas manifold was essential to ensure that gas supplies could be smoothly switched over to cylinders and an uninterruptible power supply device was also included. In testing, the Precision Flow continued to function normally during gas and power switchover. The device is shown in figure 1 .

\section{Procedure}

We followed a standardised clinical protocol for stabilisation (figure 2). The mobile apparatus could normally be set up and started within $2 \mathrm{~min}$, requiring a further $2-3 \mathrm{~min}$ to reach the desired temperature of $37^{\circ} \mathrm{C}$. During this time routine checks on the resuscitation equipment were carried out. Babies were placed into a plastic bag on a resuscitaire under a radiant warmer and assessed for the presence of breathing, heart rate and activity. Nasal prongs ('premature' size, Vapotherm) were applied and a flow of 6-7 L/min was commenced (not a stepwise increase, but clinician preference for initial flows varied). Flows up to $8 \mathrm{~L} / \mathrm{min}$ were permitted by our standard guidelines. Preductal pulse and oxygen saturation monitoring was also

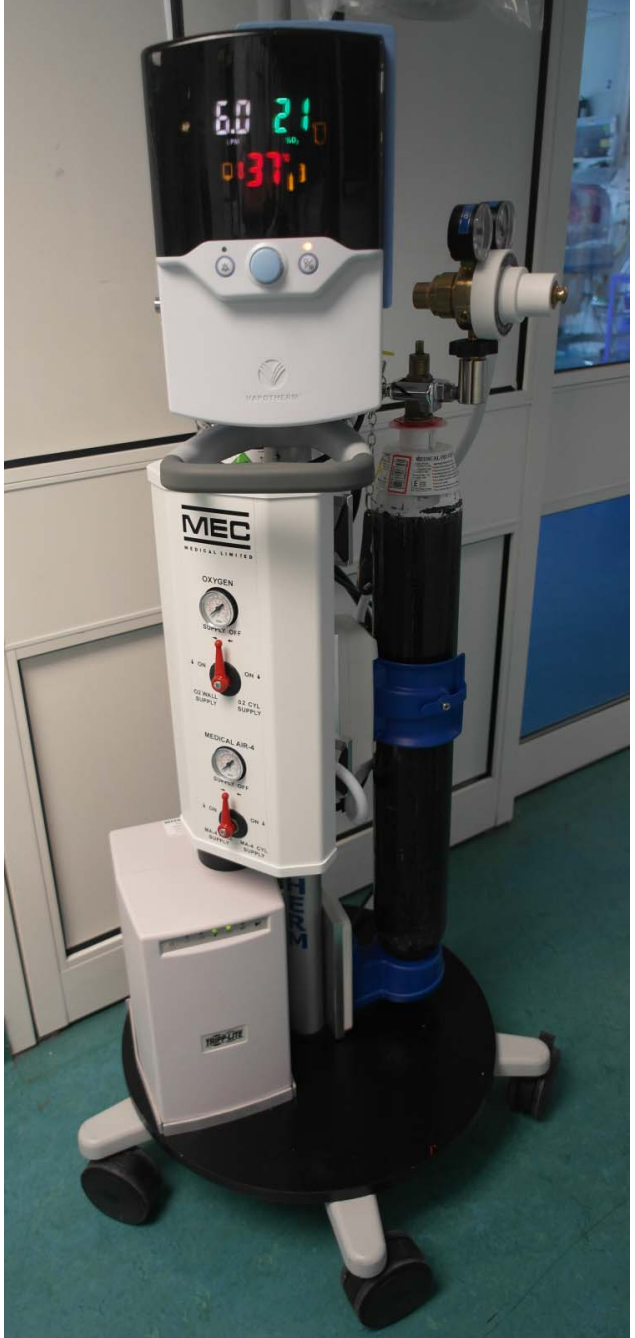

Figure 1 Mobile nasal high flow.

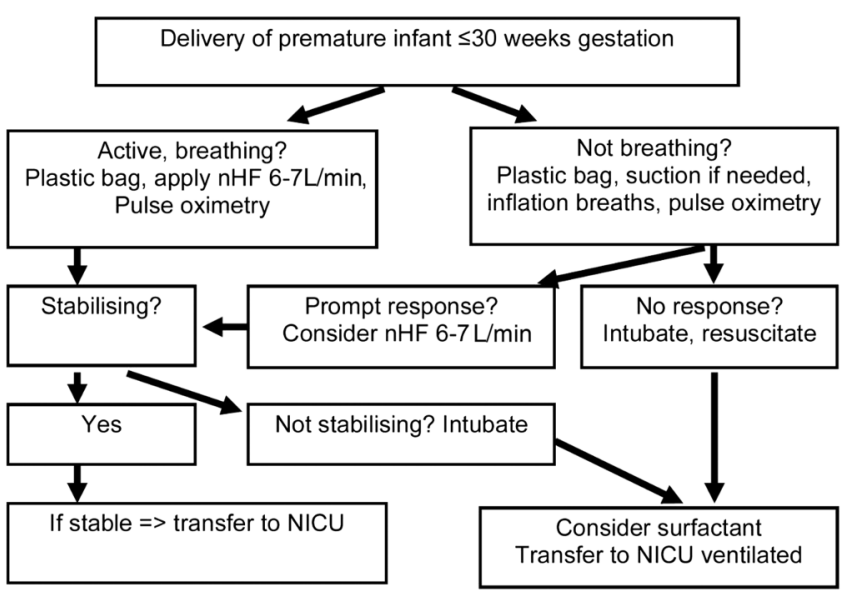

Figure 2 Protocol for stabilisation. nHF, nasal high flow; NICU, neonatal intensive care unit.

commenced using a Masimo pulse oximeter Rad-5V with values obtained after about $1 \mathrm{~min}$, and oxygen given at the clinician's discretion. Acceptable initial values were heart rate $>100 \mathrm{bpm}$ and $\mathrm{SaO}_{2}>40 \%$, with spontaneous respirations present (no rate defined). No other airway interventions such as suction or inflation breaths were applied. A steady increase in $\mathrm{SaO}_{2}$ was expected (approximately 10\% per minute over the next $5 \mathrm{~min}$ ) 
with $\mathrm{SaO}_{2}$ targeted to $90 \%-95 \%$ after $10 \mathrm{~min}$ if requiring supplemental oxygen. ${ }^{11} 12$ If the baby was apnoeic, five normal inflation breaths were applied according to standard guidance. ${ }^{13}$ If the baby responded promptly to initial resuscitation measures, then stabilisation on nHF could be attempted at the discretion of the senior clinician present. Babies who were persistently apnoeic and/or with low heart rate $(<100 / \mathrm{min})$ were managed conventionally, with suction, further inflation breaths, intubation with an appropriate-sized endotracheal tube and further resuscitation as determined by the attending clinician. The decision to administer surfactant in the DR was at the discretion of the clinician.

When stable, babies were transferred to the NICU (a short corridor journey which normally takes about a minute). On arrival in the NICU, the admission temperature was checked, pulse and oxygen saturations and $\mathrm{FiO}_{2}$ were noted and the baby was transferred to a humidified incubator. For babies on nHF the patient circuit was transferred from the mobile to the cotside nHF device which took about $20 \mathrm{~s}$.

Staff comments were invited, including on the ease of use of the protocol, the comfort of the baby if transferred on nHF and for documentation of parental comments.

\section{Study size}

The study was approved by the Research and Development Committee of Ashford and St. Peter's Hospitals NHS Foundation Trust and by the London-Surrey Borders Research Ethics Committee (REC). Written parental consent was obtained prior to delivery. The study was terminated after 28 babies had been enrolled in agreement with the R and D department and the REC as feasibility with successful completion of protocol in all cases had been established.

\section{RESULTS}

\section{Participants}

A total of 74 eligible babies were born during the study period and 33 consents were obtained. We enrolled 28 babies between $23+4$ and $29+6$ weeks gestation. The requirement for written informed consent prior to delivery meant that the majority of eligible babies could not be enrolled due to the unpredictable onset of premature delivery. Consent was obtained from five parents who did not subsequently deliver before 30 weeks. Mean birth weight was $876 \mathrm{~g}$ (range 456-1430 g). Twenty-two (79\%) were from singleton pregnancies. Ten patients were male (36\%) and $28(100 \%)$ had received at least one dose of antenatal steroids. Further characteristics of the study participants are shown in table 1.

\section{Outcomes}

Three babies born at 23 or 24 weeks were intubated and transferred to NICU ventilated as per protocol. Two of these (born at
23 and 24 weeks) subsequently died from necrotising enterocolitis (NEC) at days 13 and 30, respectively). Table 2 shows the outcome measures for all study babies. For the 25 babies stabilised and transferred on $\mathrm{nHF}$, the average admission temperature was $36.9^{\circ} \mathrm{C}$ (range $36.3^{\circ} \mathrm{C}-38.2^{\circ} \mathrm{C}$ ) and the mean $\mathrm{FiO}_{2}$ on admission to NICU was 0.29 (range 0.21-0.60), with nine babies (36\%) in room air on admission. Twelve babies (48\%) stabilised and transferred on nHF received rescue administration of surfactant, but only $5(42 \%)$ were still on nHF at $72 \mathrm{~h}$. They ranged from $23+4$ to $29+6$ weeks gestation. Of the 13 babies $(24+1$ to $29+5$ weeks gestation) who did not require surfactant, $11(85 \%)$ were still maintained on nHF at $72 \mathrm{~h}$. One baby (4\%) received inotropes in the first $72 \mathrm{~h}$ due to a pulmonary haemorrhage after $48 \mathrm{~h}$.

The smallest baby stabilised successfully weighed $498 \mathrm{~g}$, was still on nHF after $72 \mathrm{~h}$, and was eventually transferred back to their local hospital. Two babies developed grade 3 or 4 intraventricular haemorrhages (8\%). There was one pneumothorax after $24 \mathrm{~h}$, which occurred after the baby had been intubated and received IPPV before and after administration of surfactant (Curosurf). The air leak was drained without complications.

There were two documented technical issues with inadequate flow from the nHF device, both quickly resolved. The first was caused by pooled water in the chamber preventing the internal impeller from spinning, and once wiped dry the flow was normal. The second was due to a poor connection between the patient circuit and the humidification unit; again this was recognised and corrected.

In every case, the use of the mobile high flow according to the protocol was described by staff as being straightforward, even if the baby required conventional resuscitation and ventilation. Several staff commented that transfers of babies on $\mathrm{nHF}$ were 'easy' and, in 21/25 that the comfort of the babies was good (not recorded in 4/25). They also recorded positive comments from some parents about being able to see their baby's face and head movements.

\section{DISCUSSION}

In this study, we have demonstrated that it is feasible to use $\mathrm{nHF}$ for the stabilisation of premature babies without the need for routine intubation and/or surfactant prior to and during transfer to NICU in the majority of cases.

Staff followed the clinical protocol easily. The nHF could be set up and started quickly, delivering warmed and humidified gas within a couple of minutes. The nasal prongs were simple and quick to fit. We found the prongs could be effectively secured for transfer using the plastic clasp at the back of the head. Unlike nCPAP, there was no need to size and fit a hat to hold the nasal prongs.

Table 1 Characteristics of study participants $(n=28)$

\begin{tabular}{|c|c|c|c|c|c|}
\hline GA at delivery & N (\% total) & $\begin{array}{l}\text { Vaginal/caesarean } \\
\text { delivery }\end{array}$ & PPROM >24 h (\%) & $\begin{array}{l}\text { Intubated for } \\
\text { transfer to NICU }\end{array}$ & Surfactant in DR \\
\hline $23+0$ to $23+6$ & $1(4)$ & $1 / 0$ & $0(0)$ & 1 & 1 \\
\hline $24+0$ to $24+6$ & $3(11)$ & $2 / 1$ & $1(33)$ & 2 & 2 \\
\hline $25+0$ to $25+6$ & $6(18.5)$ & $2 / 4$ & $0(0)$ & 0 & 1 \\
\hline $26+0$ to $26+6$ & $5(18.5)$ & $2 / 3$ & $2(40)$ & 0 & 0 \\
\hline $27+0$ to $27+6$ & $5(18.5)$ & $2 / 3$ & $0(0)$ & 0 & 0 \\
\hline $28+0$ to $28+6$ & $5(18.5)$ & $1 / 4$ & $1(20)$ & 0 & 0 \\
\hline $29+0$ to $29+6$ & $3(11)$ & $1 / 2$ & $1(33)$ & 0 & 0 \\
\hline Mean GA 26+5 & $28(100)$ & $11(39 \%) / 17(61 \%)$ & $5(18)$ & $3(11 \%)$ & $4(14 \%)$ \\
\hline
\end{tabular}


Table 2 Outcomes in first $72 \mathrm{~h}$ after delivery $(\mathrm{n}=28)$

Stabilised and

transferred on nHF

Intubated in DR

Number of babies

25

3

Admission temperature

(mean, range)

Admission $\mathrm{FiO}_{2}$

(mean, range)

Surfactant

Inotropes

HF sustained for $72 \mathrm{~h}$

Pneumothorax

Pulmonary haemorrhage

IVH (grade 3 or 4)

$36.9^{\circ} \mathrm{C}\left(36.3^{\circ} \mathrm{C}-38.2^{\circ} \mathrm{C}\right)$

$37.3^{\circ} \mathrm{C}\left(36.3^{\circ} \mathrm{C}-38.4^{\circ} \mathrm{C}\right)$

$0.29(0.21-0.60)$

$0.37(0.3-0.5)$

$\mathrm{DR}$, delivery room; nHF, nasal high flow; IVH, intraventricular haemorrhage.

Transfer to NICU was straightforward, especially as staff did not have to hold masks or tubes in place on the baby's face. Babies were invariably stable on arrival in NICU; although one baby arrived requiring $60 \%$ oxygen, the mode $\mathrm{FiO}_{2}$ (nine babies) was 0.21 and the median was 0.27 . Our observations on the admission temperature (mean $36.9^{\circ} \mathrm{C}$, range $36.3^{\circ} \mathrm{C}-38.2^{\circ} \mathrm{C}$ ) appears to confirm that administration of humidified nasal gas at $37^{\circ} \mathrm{C}$ helps to maintain thermal stability. ${ }^{14}$ We believe that humidification is a key part of successful non-invasive respiratory management, and that the stabilisation or resuscitation of babies using cold, dry gases from wall or cylinder supplies does not constitute optimal management and should be routinely available.

Staff commented positively on the comfort of the babies, who sometimes opened their eyes and even lifted their heads. Showing parents their baby prior to transfer was also easy and some parents commented that they liked seeing their baby breathing by themselves after delivery.

In this pilot study, the majority of babies were transferred on $\mathrm{nHF}$, and less than half subsequently required surfactant. This compared favourably with our prestudy rate of $73 \%$ for DR intubation and surfactant administration. We noted that the need for surfactant appeared to predict a greater chance of intubation within $72 \mathrm{~h}$.

The group was clinically stable, with low requirements overall for early intubation/ventilation. The low incidence of hypotension requiring inotropes is consistent with our normal practice of early extubation to $\mathrm{nHF}$, and we suggest that the use of HF facilitates cardiovascular stability. The absence of nCPAP in our protocol reflects our long-standing use of $\mathrm{nHF}$ as a replacement for nCPAP in the postextubation management of preterm babies.

There are several limitations of this study. This was a small pilot study designed to generate the hypothesis that using $\mathrm{nHF}$ for DR stabilisation would be feasible. It was conducted in a single centre with considerable expertise in using nHF in premature babies, potentially limiting its current generalisability. Deliveries were always attended by either an experienced neonatal registrar and/or a consultant, which may have contributed to the successful outcomes for the majority of babies. The strengths of the study are that it demonstrated that the use of $\mathrm{nHF}$ is feasible as measured by short-term outcomes, and the clinical protocol is easy to follow.

We have described a cohort of premature babies in whom the use of intubation, ventilation, surfactant and inotropes is low, and the clinical outcomes describe a 'well' preterm population which helps us to understand how stable some babies can be from 25 weeks upwards.
We think that the use of nHF at birth contributed to this clinical stability. We recognise that the use of nHF as a primary therapy in preterm babies still has limited data to support it. Larger studies to determine either superiority or non-inferiority to other non-invasive methods for DR stabilisation of premature babies are now needed.

\section{CONCLUSIONS}

This was a pilot study designed to establish if it was possible to use nHF for DR stabilisation of preterm infants, and we believe it to be the first of its kind. The least 'successful' group for stabilisation were those born at 23-24 weeks gestation (as expected) and from 25 weeks upwards the stabilisation was, in this study, always successful. Our findings may be generalisable to other nHF devices provided that the humidification and flow can be rapidly delivered. In summary, preterm babies $<30$ weeks gestation can often be stabilised in the DR and transferred, in stable condition, to the NICU using nHF. Further work is ongoing to establish optimal flow rates and we continue to use this protocol in our day-to-day practice.

Contributors PR conceived and acted as main investigator for the study, analysing and interpreting data and drafting and revising the manuscript. He acts as the principal guarantor and corresponding author for the manuscript. SL, TL, TO and OE all helped to conduct the study including training nursing staff, collection of data and made critical revisions to the final manuscript. $\mathrm{NH}$ provided clinical support and training for the nursing staff, designed the data collection forms, collected and analysed data and contributed to the final manuscript. All authors have agreed to be accountable for all aspects of the work.

Competing interests None declared.

Ethics approval NRES Committee London-Surrey Borders.

Provenance and peer review Not commissioned; externally peer reviewed.

\section{REFERENCES}

1 Wyllie J, Bruinenberg J, Roehr CC, et al. European resuscitation council guidelines for resuscitation 2015: section 7. Resuscitation and support of transition of babies at birth. Resuscitation 2015;95:249-62.

2 Sweet DG, Carnielli V, Greisen G, et al. European consensus guidelines on the management of neonatal respiratory distress syndrome in preterm infants-2013 update. Neonatology 2013;103:353-68.

3 Finer NN, Carlo WA, Walsh MC, et al., SUPPORT Study Group of the Eunice Kennedy Shriver NICHD Neonatal Research Network. Early CPAP versus surfactant in extremely preterm infants. N Engl J Med 2010;362:1970-9.

4 Schmölzer GM, Kumar M, Pichler G, et al. Non-invasive versus invasive respiratory support in preterm infants at birth: systematic review and meta-analysis. BMJ 2013:347:f5980.

5 Rojas-Reyes MX, Morley CJ, Soll R. Prophylactic versus selective use of surfactant in preventing morbidity and mortality in preterm infants. Cochrane Database Syst Rev 2012;3:CD000510.

6 Yoder BA, Stoddard RA, Li M, et al. Heated, humidified high-flow nasal cannula versus nasal CPAP for respiratory support in neonates. Pediatrics 2013;131: e1482-90.

7 Collins $\mathrm{CL}$, Holberton JR, Barfield C, et al. A randomized controlled trial to compare heated humidified high-flow nasal cannulae with nasal continuous positive airway pressure postextubation in premature infants. J Pediatr 2013;162:949-54.e1.

8 Manley BJ, Owen LS, Doyle LW, et al. High-flow nasal cannulae in very preterm infants after extubation. N Engl J Med 2013;369:1425-33.

9 Collins CL, Barfield C, Horne RSC, et al. A comparison of nasal trauma in preterm infants extubated to either heated humidified high-flow nasal cannulae or nasal continuous positive airway pressure. Eur J Pediatr 2014;173:181-6.

10 Oxford Dictionaries Online Oxford University Press. http://www.oxforddictionaries. com/ (accessed 5 Apr 2015).

11 Dawson JA, Kamlin COF, Vento $M$, et al. Defining the reference range for oxygen saturation for infants after birth. Pediatrics 2010;125:e1340-7.

12 The BOOST II United Kingdom, Australia, and New Zealand Collaborative Groups. Oxygen saturation and outcomes in preterm infants. $N$ Engl J Med 2013;368:2094-104.

13 Newborn Life Support. Resuscitation at birth. 3rd edn. UK: Resuscitation Council, 2011.

14 Meyer MP, Hou D, Ishrar NN, et al. Initial respiratory support with cold, dry gas versus heated humidified gas and admission temperature of preterm infants. J Pediatr 2015;166:245-50. 